\title{
Stimme schonen und Schleimhäute befeuchten
}

— Ihrer Stimme messen die Deutschen eine große Bedeutung bei: Laut einer repräsentativen Umfrage im Auftrag von Engelhard Arzneimittel sind die Befragten vor allem beruflich (67\%) sehr stark bzw. stark auf sie angewiesen. Aber auch im Privatleben ist die Stimme unverzichtbar, sagen 7 von 10 Personen. Mehr als 70\% der Befragten sind von Stimmbeschwerden wie Heiserkeit, Räuspern oder Stimmbandreizungen betroffen, die meist aus einer hohen und intensiven Sprechbelastung resultieren.

Eine hohe und intensive Sprechbelastung oder ein falscher Gebrauch der Stimme können schädliche Folgen für Schleimhäute und Stimmbänder haben: Denn wenn der Sekretfilm auf der Mund- und Rachenschleimhaut versiegt und die Stimmbänder gereizt sind, können sich funktionelle Stimmstörungen einstellen. Die Heiserkeit ist dabei das hörbare Symptom. Die weiteren Beschwerden reichen von Halskratzen und Räusperzwang über ein Trockenheitsgefühl in Mund, Hals und Rachen bis hin zu Stimmbandreizungen und einem möglichen Stimmverlust. Oftmals sind diese Symptome so belastend, dass sie sich auf das Umfeld und das Wohlbefinden der Betroffenen auswirken können: Jeder Zweite ist dann genervt, 34\% fühlen sich im Berufsalltag eingeschränkt.
Zur Besserung der Stimmbeschwerden versuchen die Betroffenen, mehr zu trinken $(64,1 \%)$ und räuspern sich öfter $(28,6 \%)-$ letzeres ist eine Maßnahme, von der der Logopäde und Stimmtrainer Thomas Niemann abrät: „Beim Räuspern werden die Stimmlippen stark zusammengedrückt, das kann zu Reizungen des feinen Gewebes führen. Besser leicht husten, gähnen oder etwas trinken." Er empfiehlt, im Akutfall die Stimme zu schonen sowie ökonomisch, d.h. langsam und deutlich zu sprechen und dabei immer mal wieder Pausen einzulegen. Zudem sollte alles, was die Schleimhäute zusätzlich reizt, zum Beispiel Rauchen, Alkohol oder trockene Raumluft, vermieden werden.

Die trockenen und gereizten Schleimhäute sollten bei der Regeneration unterstützt werden. Eine regelmäßige Flüssigkeitszufuhr durch das Trinken von Wasser, Saft oder Tee ist essenziell und ein guter erster Schritt. Zusätzlich sollte auf eine nachhaltige Schleimhautbefeuchtung geachtet werden. Als wirksam und gut verträglich hat sich der Einsatz von isla ${ }^{\circledR}$ med hydro+ Milde Kirsche mit einer Kombination aus dem Spezialextrakt aus Isländisch Moos, Hyaluronsäure und einem vorgequollenen Hydrogel-Komplex erwiesen. Die drei Komponenten schützen die Schleimhäute und stellen eine intensive Befeuchtung sicher, Stimmbeschwerden und Stimmbandreizungen werden so effektiv wie nachhaltig gelindert.

red

Nach Informationen von Engelhard Arzneimittel

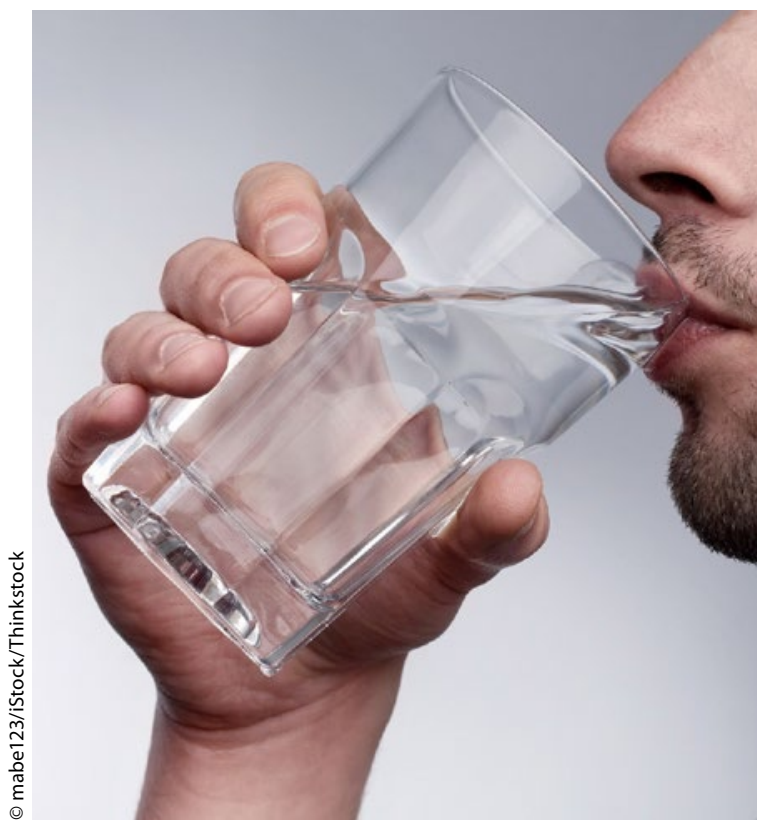

Ausreichend trinken kann helfen, trockene Schleimhäute zu befeuchten.

\section{Neues Gerät zur Impedanzmessung}

_ GN Otometrics gibt die Markteinführung seines Impedanzgerätes Madsen ${ }^{\circledR}$ Zodiac bekannt. Das neuartige Gerät bringt ein noch höheres Maß an Kontrolle, Zuverlässigkeit und Effizienz - bei seiner Entwicklung wurde zudem von Beginn an besonderer Wert auf die Sondentechnologie gelegt, so der Hersteller. „Es war unser erklärtes Ziel, ein besonders reaktionsschnelles, effizientes und zuverlässiges Impedanzgerät zu bauen" sagt Clément Sanchez, Audiologe und Produktmanager bei Otometrics. Bei den Gerätedetails fällt unter anderem die durchdachte Ergonomie der Sonden auf, ebenso wie das intuitive Interface, die robuste, benutzerfreundliche Pumpe, die problemlos zu reinigenden Oberflächen und das integrierte Workflowsystem.

Es stehen drei Sonden zur Verfügung: eine Schnelltestsonde für das Screening sowie die diagnostischen Sonden "Classic" und "Comfort" für erweiterte Tests. Die Sonden sind leicht, ergonomisch geformt und problemlos zu bedienen - so kann sie der Anwender schneller und stabiler platzieren und eine noch zuverlässigere Abdichtung erreichen. Als einziges Impedanzgerät bietet das Madsen ${ }^{\circledast}$ Zodiac die Möglichkeit des Einsatzes zweier verschiedener Sonden. Dies erlaubt es dem Anwender, die ScreeningSonde und die diagnostische Sonde gleichzeitig anzuschließen, und zwar über die gesamte Untersuchung hinweg. Darüber hinaus zeigen die Leucht- und Audioanzeigen auf der Sonde an, ob die Abdichtung vorhanden ist, welches Ohr gerade getestet wird und wann der jeweilige Test beginnt bzw. endet.

Das MADSEN Zodiac bietet ein eigenes Impedanzmodul das auf der OTOsuite $^{\oplus}$ -
Software basiert - der universellen Benutzeroberfläche für alle Otometrics-Hörtests und -Hörsystemanpassungen. Dank der flachen Menüstruktur und der bedienerfreundlichen Software wird das Arbeiten und Manövrieren mit dem System enorm vereinfacht. In nur einem unkomplizierten System kann in den Bereichen Impedanz, Untersuchung, $\mathrm{OAE}$, Video-Otoskopie und Anpassung hocheffizient gearbeitet werden. Die flexiblen Datenverwaltungsoptionen ermöglichen das Zusammenführen der Daten von einem oder mehreren Geräten zur Erstellung professioneller, benutzerdefinierter Berichte. Das neue Impedanzgerät ist in drei Ausführungen erhältlich: "Quick Check”, „Diagnostic" und "Clinical", sowie als Stand-Alone oder PC-basierte Version, wobei letztere für die Touchscreen-Bedienung optimiert ist.

Nach Informationen von GN Otometrics 